\title{
On Harmonic Functions Defined by Derivative Operator
}

\author{
K. Al-Shaqsi and M. Darus \\ School of Mathematical Sciences, Faculty of Science and Technology, Universiti Kebangsaan Malaysia, \\ Bangi 43600 Selangor D. Ehsan, Malaysia
}

Correspondence should be addressed to M. Darus, maslina@pkrisc.cc.ukm.my

Received 16 September 2007; Revised 20 November 2007; Accepted 26 November 2007

Recommended by Vijay Gupta

Let $\mathcal{S}_{\mathfrak{l}}$ denote the class of functions $f=h+\bar{g}$ that are harmonic univalent and sense-preserving in the unit disk $\mathbb{U}=\{z:|z|<1\}$, where $h(z)=z+\sum_{k=2}^{\infty} a_{k} z^{k}, g(z)=\sum_{k=1}^{\infty} b_{k} z^{k}\left(\left|b_{1}\right|<\right.$ $1)$. In this paper, we introduce the class $M_{\mathscr{l}}(n, \lambda, \alpha)$ of functions $f=h+\bar{g}$ which are harmonic in $\mathbb{U}$. A sufficient coefficient of this class is determined. It is shown that this coefficient bound is also necessary for the class $M_{\overline{\mathscr{d}}}(n, \lambda, \alpha)$ if $f_{n}(z)=h+\overline{g_{n}} \in M_{\mathscr{d}}(n, \lambda, \alpha)$, where $h(z)=z-\sum_{k=2}^{\infty}\left|a_{k}\right| z^{k}, g_{n}(z)=$ $(-1)^{n} \sum_{k=1}^{\infty}\left|b_{k}\right| z^{k}$ and $n \in \mathbb{N}_{0}$. Coefficient conditions, such as distortion bounds, convolution conditions, convex combination, extreme points, and neighborhood for the class $M_{\overline{d \ell}}(n, \lambda, \alpha)$, are obtained.

Copyright $(2008$ K. Al-Shaqsi and M. Darus. This is an open access article distributed under the Creative Commons Attribution License, which permits unrestricted use, distribution, and reproduction in any medium, provided the original work is properly cited.

\section{Introduction}

A continuous function $f=u+i v$ is a complex-valued harmonic function in a complex domain $\mathbb{C}$ if both $u$ and $v$ are real harmonic in $\mathbb{C}$. In any simply connected domain $\boldsymbol{\Phi} \mathbb{C}$, we can write $f=h+\bar{g}$, where $h$ and $g$ are analytic in $\Phi$. We call $h$ the analytic part and $g$ the coanalytic part of $f$. A necessary and sufficient condition for $f$ to be locally univalent and sense-preserving in $\nexists$ is that $\left|h^{\prime}(z)\right|>\left|g^{\prime}(z)\right|$ in $\Phi$; see [2].

Denote by $\mathcal{S}_{\mathscr{l}}$ the class of functions $f=h+\bar{g}$ that are harmonic, univalent, and sensepreserving in the unit disk $\mathbb{U}=\{z:|z|<1\}$ for which $f(0)=h(0)=f_{z}(0)-1=0$. Then for $f=h+\bar{g} \in \mathcal{S}_{\mathscr{H}}$, we may express the analytic functions $h$ and $g$ as

$$
h(z)=z+\sum_{k=2}^{\infty} a_{k} z^{k}, \quad g(z)=\sum_{k=1}^{\infty} b_{k} z^{k}, \quad\left|b_{1}\right|<1 .
$$


Observe that $\mathcal{S}_{d \ell}$ reduces to $\mathcal{S}$, the class of normalized univalent analytic functions, if the coanalytic part of $f$ is zero. Also, denote by $S_{\mathscr{d}}^{*}$ the subclasses of $\mathcal{S}_{\mathscr{d}}$ consisting of functions $f$ that map $\mathbb{U}$ onto starlike domain.

For $f=h+\bar{g}$ given by (1.1), we define the derivative operator introduced by authors (see [1]) of $f$ as

$$
\boldsymbol{\Phi}_{\lambda}^{n} f(z)=\boldsymbol{\Phi}_{\lambda}^{n} h(z)+(-1)^{n} \overline{\boldsymbol{\Phi}_{\lambda}^{n} g(z)}, \quad n, \lambda \in \mathbb{N}_{0}=\mathbb{N} \cup\{0\}, z \in \mathbb{U},
$$

where $\boldsymbol{\Phi}_{\lambda}^{n} h(z)=z+\sum_{k=2}^{\infty} k^{n} C(\lambda, k) a_{k} z^{k}, \Phi_{\lambda}^{n} g(z)=\sum_{k=1}^{\infty} k^{n} C(\lambda, k) b_{k} z^{k}$, and $C(\lambda, k)=\left(\begin{array}{c}k+\lambda-1 \\ \lambda\end{array}\right)$.

We let $M_{\mathscr{l}}(n, \lambda, \alpha)$ denote the family of harmonic functions $f$ of the form (1.1) such that

$$
\operatorname{Re}\left\{\frac{\Phi_{\lambda}^{n+1} f(z)}{\boldsymbol{\Phi}_{\lambda}^{n} f(z)}\right\}>\alpha, \quad 0 \leq \alpha<1,
$$

where $\Phi_{\lambda}^{n} f$ is defined by (1.2).

If the coanalytic part of $f=h+\bar{g}$ is identically zero, then the class $M_{\mathscr{H}}(n, \lambda, \alpha)$ turns out to be the class $R_{\lambda}^{n}(\alpha)$ introduced by Al-Shaqsi and Darus [1] for the analytic case.

Let $M_{\overline{\mathscr{d}}}(n, \lambda, \alpha)$ denote that the subclass of $M_{\mathscr{H}}(n, \lambda, \alpha)$ consists of harmonic functions $f_{n}=h+\overline{g_{n}}$ such that $h$ and $g_{n}$ are of the form

$$
h(z)=z-\sum_{k=2}^{\infty}\left|a_{k}\right| z^{k}, \quad g_{n}(z)=(-1)^{n} \sum_{k=1}^{\infty}\left|b_{k}\right| z^{k} .
$$

It is clear that the class $M_{\mathscr{H}}(n, \lambda, \alpha)$ includes a variety of well-known subclasses of $\mathcal{S}_{\mathscr{H}}$. For example, $M_{\mathscr{H}}(0,0, \alpha) \equiv S_{\mathscr{d}}^{*}(\alpha)$ is the class of sense-preserving, harmonic, univalent functions $f$ which are starlike of order $\alpha$ in $\mathbb{U}$, that is, $(\partial / \partial \theta)\left\{\arg \left(f\left(\operatorname{re}^{i \theta}\right)\right)\right\}>\alpha$, and $M_{\mathscr{l}}(1,0, \alpha) \equiv$ $M_{\mathscr{H}}(0,1, \alpha) \equiv \mathscr{H} \mathcal{K}(\alpha)$ is the class of sense-preserving, harmonic, univalent functions $f$ which are convex of order $\alpha$ in $\mathbb{U}$, that is, $(\partial / \partial \theta)\left\{\arg \left((\partial / \partial \theta) f\left(\mathrm{re}^{i \theta}\right)\right)\right\}>\alpha$. Note that the classes $S_{\mathscr{d}}^{*}$ and $\mathscr{H} \mathcal{K}(\alpha)$ were introduced and studied by Jahangiri [3]. Also we notice that the class $M_{\bar{d}}(n, 0, \alpha)$ is the class of Salagean-type harmonic univalent functions introduced by Jahangiri et al. [4]; and $M_{\overline{d d}}(0, \lambda, \alpha)$ is the class of Ruscheweyh-type harmonic univalent functions studied by Murugusundaramoorthy and Vijaya [5].

In 1984, Clunie and Sheil-Small [2] investigated the class $\mathcal{S}_{\mathfrak{d}}$ as well as its geometric subclasses and obtained some coefficient bounds. Since then, there has been several related papers on $\mathcal{S}_{\mathfrak{d}}$ and its subclasses such that Silverman [6], Silverman and Silvia [7], and Jahangiri [3, 8] studied the harmonic univalent functions. Jahangiri and Silverman [9] prove the following theorem.

Theorem 1.1. Let $f=h+\bar{g}$ given by (1.1). If

$$
\sum_{k=2}^{\infty} k\left(\left|a_{k}\right|+\left|b_{k}\right|\right) \leq 1-\left|b_{1}\right|
$$

then $f$ is sense-preserving, harmonic, and univalent in $\mathbb{U}$ and $f \in S_{\mathscr{d}}^{*}$ consists of functions in $\mathcal{S}_{\mathfrak{d}}$ which are starlike in $\mathbb{U}$.

The condition (1.5) is also necessary if $f \in \tau H \equiv M_{\overline{d \ell}}(0,0,0)$.

In this paper, we will give sufficient condition for functions $f=h+\bar{g}$, where $h$ and $g$ are given by (1.1) to be in the class $M_{\mathscr{l}}(n, \lambda, \alpha)$; and it is shown that this coefficient condition is 
also necessary for functions in the class $M_{\bar{d}}(n, \lambda, \alpha)$. Also, we obtain distortion theorems and characterize the extreme points for functions in $M_{\overline{\mathscr{l}}}(n, \lambda, \alpha)$. Closure theorems and application of neighborhood are also obtained.

\section{Coefficient bounds}

We begin with a sufficient coefficient condition for functions in $M_{\mathscr{l}}(n, \lambda, \alpha)$.

Theorem 2.1. Let $f=h+\bar{g}$ be given by (1.1). If

$$
\sum_{k=1}^{\infty}\left[(k-\alpha)\left|a_{k}\right|+(k+\alpha)\left|b_{k}\right|\right] k^{n} C(\lambda, k) \leq 2(1-\alpha),
$$

where $a_{1}=1, n, \lambda \in \mathbb{N}_{0}, C(\lambda, k)=(\underset{\lambda}{k+\lambda-1})$, and $0 \leq \alpha<1$, then $f$ is sense-preserving, harmonic, univalent in $\mathbb{U}$, and $f \in M_{\mathscr{H}}(n, \lambda, \alpha)$.

Proof. If $z_{1} \neq z_{2}$, then

$$
\begin{aligned}
\left|\frac{f\left(z_{1}\right)-f\left(z_{2}\right)}{h\left(z_{1}\right)-h\left(z_{2}\right)}\right| & \geq 1-\left|\frac{g\left(z_{1}\right)-g\left(z_{2}\right)}{h\left(z_{1}\right)-h\left(z_{2}\right)}\right| \\
& =1-\left|\frac{\sum_{k=1}^{\infty} b_{k}\left(z_{1}^{k}-z_{2}^{k}\right)}{\left(z_{1}-z_{2}\right)+\sum_{k=2}^{\infty} a_{k}\left(z_{1}^{k}-z_{2}^{k}\right)}\right| \\
& >1-\frac{\sum_{k=1}^{\infty} k\left|b_{k}\right|}{1-\sum_{k=2}^{\infty} k\left|a_{k}\right|} \\
& \geq 1-\frac{\sum_{k=1}^{\infty}\left((k+\alpha) k^{n} C(\lambda, k) /(1-\alpha)\right)\left|b_{k}\right|}{1-\sum_{k=2}^{\infty}\left((k-\alpha) k^{n} C(\lambda, k) /(1-\alpha)\right)\left|a_{k}\right|} \geq 0,
\end{aligned}
$$

which proves univalence. Note that $f$ is sense-preserving in $\mathbb{U}$. This is because

$$
\begin{aligned}
\left|h^{\prime}(z)\right| & \geq 1-\sum_{k=2}^{\infty} k\left|a_{k}\right||z|^{k-1} \\
& >1-\sum_{k=2}^{\infty} \frac{(k-\alpha) k^{n} C(\lambda, k)}{1-\alpha}\left|a_{k}\right| \\
& \geq \sum_{k=1}^{\infty} \frac{(k+\alpha) k^{n} C(\lambda, k)}{1-\alpha}\left|b_{k}\right| \\
& >\sum_{k=1}^{\infty} \frac{(k+\alpha) k^{n} C(\lambda, k)}{1-\alpha}\left|b_{k}\right||z|^{k-1} \geq \sum_{k=1}^{\infty} k\left|b_{k}\right||z|^{k-1} \geq\left|g^{\prime}(z)\right| .
\end{aligned}
$$

Using the fact that Rew $>\alpha$ if and only if $|1-\alpha+w| \geq|1+\alpha-w|$, it suffices to show that

$$
\left|(1-\alpha) \Phi_{\lambda}^{n} f(z)+\Phi_{\lambda}^{n+1} f(z)\right|-\left|(1+\alpha) \Phi_{\lambda}^{n} f(z)-\Phi_{\lambda}^{n+1} f(z)\right| \geq 0
$$


Substituting $\Phi_{\lambda}^{n} f(z)$ in (2.4) yields, by (2.1), we obtain

$$
\begin{aligned}
\left|(1-\alpha) \Phi_{\lambda}^{n} f(z)+\Phi_{\lambda}^{n+1} f(z)\right|-\left|(1+\alpha) \Phi_{\lambda}^{n} f(z)-\Phi_{\lambda}^{n+1} f(z)\right| \\
=\left|(2-\alpha) z+\sum_{k=2}^{\infty}(k+1-\alpha) k^{n} C(\lambda, k) a_{k} z^{k}-(-1)^{n} \sum_{k=1}^{\infty}(k-1+\alpha) k^{n} C(\lambda, k) \overline{b_{k} z^{k}}\right| \\
\quad-\left|-\alpha z+\sum_{k=2}^{\infty}(k-1-\alpha) k^{n} C(\lambda, k) a_{k} z^{k}-(-1)^{n} \sum_{k=1}^{\infty}(k+1+\alpha) k^{n} C(\lambda, k) \overline{b_{k} z^{k}}\right| \\
\geq 2(1-\alpha)|z|\left\{1-\sum_{k=2}^{\infty} \frac{(k-\alpha) k^{n} C(\lambda, k)}{1-\alpha}\left|a_{k}\right||z|^{k-1} \sum_{k=1}^{\infty} \frac{(k+\alpha) k^{n} C(\lambda, k)}{1-\alpha}\left|b_{k}\right||z|^{k-1}\right\} \\
\geq 2(1-\alpha)\left\{1-\sum_{k=2}^{\infty} \frac{(k-\alpha) k^{n} C(\lambda, k)}{1-\alpha}\left|a_{k}\right|-\sum_{k=1}^{\infty} \frac{(k+\alpha) k^{n} C(\lambda, k)}{1-\alpha}\left|b_{k}\right|\right\} .
\end{aligned}
$$

This last expression is nonnegative by (2.1), and so the proof is complete.

The harmonic function

$$
f(z)=z+\sum_{k=2}^{\infty} \frac{1-\alpha}{(k-\alpha) k^{n} C(\lambda, k)} x_{k} z^{k}+\sum_{k=1}^{\infty} \frac{1-\alpha}{(k+\alpha) k^{n} C(\lambda, k)} \overline{y_{k} z^{k}}
$$

where $n, \lambda \in \mathbb{N}_{0}$ and $\sum_{k=2}^{\infty}\left|x_{k}\right|+\sum_{k=1}^{\infty}\left|y_{k}\right|=1$ show that the coefficient bound given by (2.1) is sharp. The functions of the form (2.6) are in $M_{d \ell}(n, \lambda, \alpha)$ because

$$
\sum_{k=1}^{\infty}\left[\frac{k-\alpha}{1-\alpha}\left|a_{k}\right|+\frac{k+\alpha}{1-\alpha}\left|b_{k}\right|\right] k^{n} C(\lambda, k)=1+\sum_{k=2}^{\infty}\left|x_{k}\right|+\sum_{k=1}^{\infty}\left|y_{k}\right|=2
$$

In the following theorem, it is shown that the condition (2.1) is also necessary for functions $f_{n}=h+\overline{g_{n}}$, where $h$ and $g_{n}$ are of the form (1.4).

Theorem 2.2. Let $f_{n}=h+\overline{g_{n}}$ be given by (1.4). Then $f_{n} \in M_{\overline{\mathscr{d}}}(n, \lambda, \alpha)$ if and only if

$$
\sum_{k=1}^{\infty}\left[(k-\alpha)\left|a_{k}\right|+(k+\alpha)\left|b_{k}\right|\right] k^{n} C(\lambda, k) \leq 2(1-\alpha),
$$

where $a_{1}=1, n, \lambda \in \mathbb{N}_{0}, C(\lambda, k)=\left(\begin{array}{c}k+\lambda-1 \\ \lambda\end{array}\right)$, and $0 \leq \alpha<1$.

Proof. Since $M_{\overline{\mathscr{l}}}(n, \lambda, \alpha) \subset M_{\mathscr{l}}(n, \lambda, \alpha)$, we only need to prove the "if and only if" part of the theorem. To this end, for functions $f_{n}$ of the form (1.4), we notice that the condition (1.3) is equivalent to

$$
\operatorname{Re}\left\{\frac{(1-\alpha) z-\sum_{k=2}^{\infty}(k-\alpha) k^{n} C(\lambda, k) a_{k} z^{k}-(-1)^{2 n} \sum_{k=1}^{\infty}(k+\alpha) k^{n} C(\lambda, k) b_{k} \overline{z^{k}}}{z-\sum_{k=2}^{\infty} k^{n} C(\lambda, k) a_{k} z^{k}+(-1)^{2 n} \sum_{k=1}^{\infty} k^{n} C(\lambda, k) b_{k} \overline{z^{k}}}\right\} \geq 0
$$


The above required condition (2.9) must hold for all values of $z$ in $\mathbb{U}$. Upon choosing the values of $z$ on the positive real axis, where $0 \leq z=r<1$, we must have

$$
\frac{1-\alpha-\sum_{k=2}^{\infty}(k-\alpha) k^{n} C(\lambda, k) a_{k} r^{k-1}-\sum_{k=1}^{\infty}(k+\alpha) k^{n} C(\lambda, k) b_{k} r^{k-1}}{1-\sum_{k=2}^{\infty} k^{n} C(\lambda, k) a_{k} r^{k-1}+\sum_{k=1}^{\infty} k^{n} C(\lambda, k) \overline{b_{k} r^{k-1}}} \geq 0 .
$$

If the condition (2.8) does not hold, then the numerator in (2.10) is negative for $r$ sufficiently close to 1 . Hence there exist $z_{0}=r_{0}$ in $(0,1)$ for which the quotient in $(2.8)$ is negative. This contradicts the required condition for $f_{n} \in M_{\overline{d \ell}}(n, \lambda, \alpha)$ and so the proof is complete.

\section{Distortion bounds}

In this section, we will obtain distortion bounds for functions in $M_{\bar{d}}(n, \lambda, \alpha)$.

Theorem 3.1. Let $f_{n} \in M_{\overline{\mathscr{d}}}(n, \lambda, \alpha)$. Then for $|z|=r<1$, one has

$$
\begin{aligned}
& \left|f_{n}(z)\right| \leq\left(1+\left|b_{1}\right|\right) r+\frac{1}{2^{n}(\lambda+1)}\left(\frac{1-\alpha}{2-\alpha}-\frac{1+\alpha}{2-\alpha}\left|b_{1}\right|\right) r^{2}, \\
& \left|f_{n}(z)\right| \geq\left(1-\left|b_{1}\right|\right) r-\frac{1}{2^{n}(\lambda+1)}\left(\frac{1-\alpha}{2-\alpha}-\frac{1+\alpha}{2-\alpha}\left|b_{1}\right|\right) r^{2} .
\end{aligned}
$$

Proof. We only prove the left-hand inequality. The proof for the right-hand inequality is similar and will be omitted. Let $f_{n} \in M_{\overline{\mathscr{d}}}(n, \lambda, \alpha)$. Taking the absolute value of $f_{n}$, we obtain

$$
\begin{aligned}
\left|f_{n}(z)\right| & =\left|z-\sum_{k=2}^{\infty} a_{k} z^{k}+(-1)^{n} \sum_{k=1}^{\infty} b_{k} \bar{z}^{k}\right| \\
& \geq\left(1-\left|b_{1}\right|\right) r-\sum_{k=2}^{\infty}\left(\left|a_{k}\right|+\left|b_{k}\right|\right) r^{k} \\
& \geq\left(1-\left|b_{1}\right|\right) r-r^{2} \sum_{k=2}^{\infty}\left(\left|a_{k}\right|+\left|b_{k}\right|\right) \\
& \geq\left(1-\left|b_{1}\right|\right) r-\frac{1-\alpha}{(2-\alpha) 2^{n}(\lambda+1)}\left(\sum_{k=2}^{\infty} \frac{(2-\alpha) 2^{n}(\lambda+1)}{1-\alpha}\left|a_{k}\right|+\frac{(2-\alpha) 2^{n}(\lambda+1)}{1-\alpha}\left|b_{k}\right|\right) r^{2} \\
& \geq\left(1-\left|b_{1}\right|\right) r-\frac{1-\alpha}{(2-\alpha) 2^{n}(\lambda+1)}\left(\sum_{k=2}^{\infty} \frac{(k-\alpha) k^{n} C(\lambda, k)}{1-\alpha}\left|a_{k}\right|+\frac{(k+\alpha) k^{n} C(\lambda, k)}{1-\alpha}\left|b_{k}\right|\right) r^{2} \\
& \geq\left(1-\left|b_{1}\right|\right) r-\frac{1-\alpha}{(2-\alpha) 2^{n}(\lambda+1)}\left(1-\frac{1+\alpha}{1-\alpha}\left|b_{1}\right|\right) r^{2} .
\end{aligned}
$$

The functions

$$
\begin{aligned}
& f(z)=z+\left|b_{1}\right| \bar{z}+\frac{1}{2^{n}(\lambda+1)}\left(\frac{1-\alpha}{2-\alpha}-\frac{1+\alpha}{2-\alpha}\left|b_{1}\right|\right) \bar{z}^{2} \\
& f(z)=\left(1-\left|b_{1}\right|\right) z-\frac{1}{2^{n}(\lambda+1)}\left(\frac{1-\alpha}{2-\alpha}-\frac{1+\alpha}{2-\alpha}\left|b_{1}\right|\right) z^{2}
\end{aligned}
$$

for $\left|b_{1}\right| \leq(1-\alpha) /(1+\alpha)$ show that the bounds given in Theorem 3.1 are sharp. 
The following covering result follows from the left-hand inequality in Theorem 3.1.

Corollary 3.2. If the function $f_{n}=h+\overline{g_{n}}$, where $h$ and $g$ given by $(1.4)$ are in $M_{\overline{d e}}(n, \lambda, \alpha)$, then

$$
\left\{w:|w|<\frac{2^{n+1}(\lambda+1)-1-\left(2^{n}(\lambda+1)-1\right) \alpha}{2^{n}(\lambda+1)(2-\alpha)}-\frac{2^{n+1}(\lambda+1)-1-\left(2^{n}(\lambda+1)+1\right) \alpha}{2^{n}(\lambda+1)(2-\alpha)}\left|b_{1}\right|\right\} \subset f_{n}(\mathbb{U}) .
$$

\section{Convolution, convex combination, and extreme points}

In this section, we show that the class $M_{\overline{d \ell}}(n, \lambda, \alpha)$ is invariant under convolution and convex combination of its member.

For harmonic functions $f_{n}(z)=z-\sum_{k=2}^{\infty} a_{k} z^{k}+(-1)^{n} \sum_{k=1}^{\infty} b_{k} \bar{z}^{k}$ and $F_{n}(z)=z-$ $\sum_{k=2}^{\infty} A_{k} z^{k}+(-1)^{n} \sum_{k=1}^{\infty} B_{k} \bar{z}^{k}$, the convolution of $f_{n}$ and $F_{n}$ is given by

$$
\left(f_{n} * F_{n}\right)(z)=f_{n}(z) * F_{n}(z)=z-\sum_{k=2}^{\infty} a_{k} A_{k} z^{k}+(-1)^{n} \sum_{k=1}^{\infty} b_{k} B_{k} \bar{z}^{k} .
$$

Theorem 4.1. For $0 \leq \beta \leq \alpha<1$, let $f_{n} \in M_{\overline{d d}}(n, \lambda, \alpha)$ and $F_{n} \in M_{\bar{d}}(n, \lambda, \beta)$. Then $f_{n} * F_{n}$ $\in M_{\overline{\mathscr{d}}}(n, \lambda, \alpha) \subset M_{\overline{\mathscr{d}}}(n, \lambda, \beta)$.

Proof. We wish to show that the coefficients of $f_{n} * F_{n}$ satisfy the required condition given in Theorem 2.2. For $F_{n} \in M_{\overline{\mathscr{d}}}(n, \lambda, \beta)$, we note that $\left|A_{k}\right| \leq 1$ and $\left|B_{k}\right| \leq 1$. Now, for the convolution function $f_{n} * F_{n}$, we obtain

$$
\begin{aligned}
\sum_{k=2}^{\infty} \frac{(k-\beta) k^{n} C(\lambda, k)}{1-\beta}\left|a_{k}\right|\left|A_{k}\right|+\sum_{k=1}^{\infty} \frac{(k+\beta) k^{n} C(\lambda, k)}{1-\beta}\left|b_{k}\right|\left|B_{k}\right| \\
\quad \leq \sum_{k=2}^{\infty} \frac{(k-\beta) k^{n} C(\lambda, k)}{1-\beta}\left|a_{k}\right|+\sum_{k=1}^{\infty} \frac{(k+\beta) k^{n} C(\lambda, k)}{1-\beta}\left|b_{k}\right| \\
\quad \leq \sum_{k=2}^{\infty} \frac{(k-\alpha) k^{n} C(\lambda, k)}{1-\alpha}\left|a_{k}\right|+\sum_{k=1}^{\infty} \frac{(k+\alpha) k^{n} C(\lambda, k)}{1-\alpha}\left|b_{k}\right| \leq 1,
\end{aligned}
$$

since $0 \leq \beta \leq \alpha<1$ and $f_{n} \in M_{\overline{\mathscr{d}}}(n, \lambda, \alpha)$. Therefore $f_{n} * F_{n} \in M_{\overline{\mathscr{d}}}(n, \lambda, \alpha) \subset M_{\overline{\mathscr{d}}}(n, \lambda, \beta)$.

We now examine the convex combination of $M_{\overline{d \ell}}(n, \lambda, \alpha)$.

Let the functions $f_{n_{j}}(z)$ be defined, for $j=1,2, \ldots$, by

$$
f_{n_{j}}(z)=z-\sum_{k=2}^{\infty}\left|a_{k, j}\right| z^{k}+(-1)^{n} \sum_{k=1}^{\infty}\left|b_{k, j}\right| \bar{z}^{k}
$$

Theorem 4.2. Let the functions $f_{n_{j}}(z)$ defined by (4.3) be in the class $M_{\overline{d e}}(n, \lambda, \alpha)$ for every $j=$ $1,2, \ldots, m$. Then the functions $t_{j}(z)$ defined by

$$
t_{j}(z)=\sum_{j=1}^{m} c_{j} f_{n_{j}}(z), \quad 0 \leq c_{j} \leq 1
$$

are also in the class $M_{\overline{d e}}(n, \lambda, \alpha)$, where $\sum_{j=1}^{m} c_{j}=1$. 
Proof. According to the definition of $t_{j}$, we can write

$$
t_{j}(z)=z-\sum_{k=2}^{\infty}\left(\sum_{j=1}^{m} c_{j} a_{k, j}\right) z^{k}+(-1)^{n} \sum_{k=1}^{\infty}\left(\sum_{j=1}^{m} c_{j} b_{n, j}\right) \bar{z}^{k}
$$

Further, since $f_{n_{j}}(z)$ are in $M_{\overline{\mathscr{d}}}(n, \lambda, \alpha)$ for every $j=1,2, \ldots$, then by $(2.8)$, we have

$$
\begin{aligned}
\sum_{k=1}^{\infty}\{ & {\left.\left[(k-\alpha)\left(\sum_{j=1}^{m} c_{j}\left|a_{k, j}\right|\right)+(k+\alpha)\left(\sum_{j=1}^{m} c_{j}\left|b_{k, j}\right|\right)\right] k^{n} C(\lambda, k)\right\} } \\
= & \sum_{j=1}^{m} c_{j}\left(\sum_{k=1}^{\infty}\left[(k-\alpha)\left|a_{n, j}\right|+(k+\alpha)\left|b_{n, j}\right|\right] k^{n} C(\lambda, k)\right) \\
& \leq \sum_{j=1}^{m} c_{j} 2(1-\alpha) \leq 2(1-\alpha) .
\end{aligned}
$$

Hence the theorem follows.

Corollary 4.3. The class $M_{\overline{d e}}(n, \lambda, \alpha)$ is closed under convex linear combination.

Proof. Let the functions $f_{n_{j}}(z)(j=1,2)$ defined by $(4.1)$ be in the class $M_{\bar{d}}(n, \lambda, \alpha)$. Then the function $\Psi(z)$ defined by

$$
\Psi(z)=\mu f_{n_{1}}(z)+(1-\mu) f_{n_{2}}(z), \quad 0 \leq \mu \leq 1
$$

is in the class $M_{\overline{d \ell}}(n, \lambda, \alpha)$. Also, by taking $m=2, t_{1}=\mu$, and $t_{2}=(1-\mu)$ in Theorem 4.1, we have the corollary.

Next we determine the extreme points of closed convex hulls of $M_{\overline{\mathscr{d}}}(n, \lambda, \alpha)$ denoted by $\operatorname{clco} M_{\overline{\mathscr{d}}}(n, \lambda, \alpha)$.

Theorem 4.4. Let $f_{n}$ be given by (1.4). Then $f_{n} \in M_{\overline{d \ell}}(n, \lambda, \alpha)$ if and only if

$$
f_{n}(z)=\sum_{k=1}^{\infty}\left(X_{k} h_{k}(z)+Y_{k} g_{n_{k}}(z)\right)
$$

where $h_{1}(z)=z, h_{k}(z)=z-\left((1-\alpha) /(k-\alpha) k^{n} C(\lambda, k)\right) z^{k}, k=2,3, \ldots, g_{n_{k}}(z)=z+$ $(-1)^{n}\left((1-\alpha) /(k+\alpha) k^{n} C(\lambda, k)\right) \bar{z}^{k}, k=1,2,3, \ldots$, and $\sum_{k=1}^{\infty}\left(X_{k}+Y_{k}\right)=1, X_{k} \geq 0, Y_{k} \geq 0$. In particular, the extreme points of $M_{\overline{d e}}(n, \lambda, \alpha)$ are $\left\{h_{k}\right\}$ and $\left\{g_{n_{k}}\right\}$.

Proof. For the functions $f_{n}$ of the form (4.8), we have

$$
\begin{aligned}
f_{n}(z) & =\sum_{k=1}^{\infty}\left(X_{k} h_{k}(z)+Y_{k} g_{n_{k}}(z)\right) \\
& =\sum_{k=1}^{\infty}\left(X_{k}+Y_{k}\right) z-\sum_{k=2}^{\infty} \frac{1-\alpha}{(k-\alpha) k^{n} C(\lambda, k)} X_{k} z^{k}+(-1)^{n} \sum_{k=1}^{\infty} \frac{1-\alpha}{(k+\alpha) k^{n} C(\lambda, k)} Y_{k} \bar{z}^{k}
\end{aligned}
$$

Then

$$
\sum_{k=2}^{\infty} \frac{(k-\alpha) k^{n} C(\lambda, k)}{1-\alpha}\left|a_{k}\right|+\sum_{k=1}^{\infty} \frac{(k+\alpha) k^{n} C(\lambda, k)}{1-\alpha}\left|b_{k}\right|=\sum_{k=2}^{\infty} X_{k}+\sum_{k=1}^{\infty} Y_{k}=1-X_{1} \leq 1,
$$


and so $f_{n} \in \operatorname{clco} M_{\overline{d \ell}}(n, \lambda, \alpha)$.

Conversely, suppose that $f_{n} \in \operatorname{clco} M_{\overline{\mathscr{d}}}(n, \lambda, \alpha)$. Setting

$$
\begin{aligned}
& X_{k}=\frac{(k-\alpha) k^{n} C(\lambda, k)}{1-\alpha}\left|a_{k}\right|, \quad 0 \leq X_{k} \leq 1, k=2,3, \ldots, \\
& Y_{k}=\frac{(k+\alpha) k^{n} C(\lambda, k)}{1-\alpha}\left|b_{k}\right|, \quad 0 \leq Y_{k} \leq 1, k=1,2,3, \ldots,
\end{aligned}
$$

and $X_{1}=1-\sum_{k=2}^{\infty} X_{k}-\sum_{k=1}^{\infty} Y_{k}$. Therefore, $f_{n}$ can be written as

$$
\begin{aligned}
f_{n}(z) & =z-\sum_{k=2}^{\infty}\left|a_{k}\right| z^{k}+(-1)^{n} \sum_{k=1}^{\infty}\left|b_{k}\right| \bar{z}^{k} \\
& =z-\sum_{k=2}^{\infty} \frac{(1-\alpha) X_{k}}{(k-\alpha) k^{n} C(\lambda, k)} z^{k}+(-1)^{n} \sum_{k=1}^{\infty} \frac{(1-\alpha) Y_{k}}{(k+\alpha) k^{n} C(\lambda, k)} \bar{z}^{k} \\
& =z+\sum_{k=2}^{\infty}\left(h_{k}(z)-z\right) X_{k}+\sum_{k=1}^{\infty}\left(g_{n_{k}}(z)-z\right) Y_{k} \\
& =\sum_{k=2}^{\infty} h_{k}(z) X_{k}+\sum_{k=1}^{\infty} g_{n_{k}}(z) Y_{k}+z\left(1-\sum_{k=2}^{\infty} X_{k}-\sum_{k=1}^{\infty} Y_{k}\right) \\
& =\sum_{k=1}^{\infty}\left(h_{k}(z) X_{k}+g_{n_{k}}(z) Y_{k}\right), \text { as required. }
\end{aligned}
$$

Using Corollary 4.3 we have $\operatorname{clco} M_{\overline{\mathscr{d}}}(n, \lambda, \alpha)=M_{\overline{\mathscr{d}}}(n, \lambda, \alpha)$. Then the statement of Theorem 4.4 is really for $f \in M_{\overline{\mathscr{d}}}(n, \lambda, \alpha)$.

\section{An application of neighborhood}

In this section, we will prove that the functions in a neighborhood of $M_{\bar{d}}(n, \lambda, \alpha)$ are starlike harmonic functions.

Following [10], we defined the $\delta$-neighborhood of a function $f \in \tau H$ by

$$
\mathcal{N}_{\delta}(f)=\left\{F(z)=z-\sum_{k=2}^{\infty} A_{k} z^{k}-\sum_{k=1}^{\infty} B_{k} \bar{z}^{k}, \sum_{k=2}^{\infty} k\left[\left|a_{k}-A_{k}\right|+\left|b_{k}-B_{k}\right|\right]+\left|b_{1}-B_{1}\right| \leq \delta\right\}
$$

where $\delta>0$.

Theorem 5.1. Let

$$
\delta=\frac{(2-\alpha) 2^{n}(\lambda+1)-1+\alpha-\left((2-\alpha) 2^{n}(\lambda+1)-1-\alpha\right)\left|b_{1}\right|}{(2-\alpha) 2^{n}(\lambda+1)}
$$

Then $\mathcal{N}_{\delta}\left(M_{\overline{\mathscr{d}}}(n, \lambda, \alpha)\right) \subset \tau H$. 
Proof. Suppose $f_{n} \in M_{\overline{d d}}(n, \lambda, \alpha)$. Let $F_{n}=H+\overline{G_{n}} \in \mathcal{N}_{\delta}\left(f_{n}\right)$, where $H=z-\sum_{k=2}^{\infty} A_{k} z^{k}$ and $G_{n}=(-1)^{n} \sum_{k=1}^{\infty} B_{k} z^{k}$. We need to show that $F_{n} \in \tau H$. In other words, it suffices to show that $F_{n}$ satisfies the condition $\tau(F)=\sum_{k=2}^{\infty} k\left[\left|A_{k}\right|+\left|B_{k}\right|\right]+\left|B_{1}\right| \leq 1$. We observe that

$$
\begin{aligned}
\tau(F) & =\sum_{k=2}^{\infty} k\left[\left|A_{k}\right|+\left|B_{k}\right|\right]+\left|B_{1}\right| \\
& =\sum_{k=2}^{\infty} k\left[\left|A_{k}-a_{k}+a_{k}\right|+\left|B_{k}-b_{k}+b_{k}\right|\right]+\left|B_{1}-b_{1}+b_{1}\right| \\
& =\sum_{k=2}^{\infty} k\left[\left|A_{k}-a_{k}\right|+\left|B_{k}-b_{k}\right|\right]+\sum_{k=2}^{\infty} k\left[\left|a_{k}\right|+\left|b_{k}\right|\right]+\left|B_{1}-b_{1}\right|+\left|b_{1}\right| \\
& =\left(\sum_{k=2}^{\infty} k\left[\left|A_{k}-a_{k}\right|+\left|B_{k}-b_{k}\right|\right]+\left|B_{1}-b_{1}\right|\right)+\sum_{k=2}^{\infty} k\left[\left|a_{k}\right|+\left|b_{k}\right|\right]+\left|b_{1}\right| \\
& =\delta+\left|b_{1}\right|+\sum_{k=2}^{\infty} k\left[\left|a_{k}\right|+\left|b_{k}\right|\right] \\
& =\delta+\left|b_{1}\right|+\frac{1-\alpha}{(2-\alpha) 2^{n}(\lambda+1)} \sum_{k=2}^{\infty}\left[\frac{2-\alpha}{1-\alpha}\left|a_{k}\right|+\frac{2+\alpha}{1-\alpha}\left|b_{k}\right|\right] 2^{n}(\lambda+1) \\
& \leq \delta+\left|b_{1}\right|+\frac{1-\alpha}{(2-\alpha) 2^{n}(\lambda+1)} \sum_{k=2}^{\infty}\left[\frac{k-\alpha}{1-\alpha}\left|a_{k}\right|+\frac{k+\alpha}{1-\alpha}\left|b_{k}\right|\right] k^{n} C(\lambda, k) \\
& \leq \delta+\left|b_{1}\right|+\frac{1-\alpha}{(2-\alpha) 2^{n}(\lambda+1)}\left(1-\frac{1+\alpha}{1-\alpha}\left|b_{1}\right|\right) .
\end{aligned}
$$

Now this last expression is never greater than one if

$$
\begin{aligned}
\delta & \leq 1-\left|b_{1}\right|-\frac{1-\alpha}{(2-\alpha) 2^{n}(\lambda+1)}\left(1-\frac{1+\alpha}{1-\alpha}\left|b_{1}\right|\right) \\
& =\frac{(2-\alpha) 2^{n}(\lambda+1)-1+\alpha-\left((2-\alpha) 2^{n}(\lambda+1)-1-\alpha\right)\left|b_{1}\right|}{(2-\alpha) 2^{n}(\lambda+1)} .
\end{aligned}
$$

\section{Acknowledgment}

The work presented here was supported by Fundamental Research Grant Scheme UKM-ST-01FRGS0055-2006.

\section{References}

[1] K. Al-Shaqsi and M. Darus, "An operator defined by convolution involving the polylogarithms functions," submitted.

[2] J. Clunie and T. Sheil-Small, "Harmonic univalent functions," Annales Academiae Scientiarum Fennicae. Series A I, vol. 9, pp. 3-25, 1984.

[3] J. M. Jahangiri, "Harmonic functions starlike in the unit disk," Journal of Mathematical Analysis and Applications, vol. 235, no. 2, pp. 470-477, 1999.

[4] J. M. Jahangiri, G. Murugusundaramoorthy, and K. Vijaya, "Salagean-type harmonic univalent functions," Southwest Journal of Pure and Applied Mathematics, no. 2, pp. 77-82, 2002. 
[5] G. Murugusundaramoorthy and K. Vijaya, "On certain classes of harmonic functions involving Ruscheweyh derivatives," Bulletin of the Calcutta Mathematical Society, vol. 96, no. 2, pp. 99-108, 2004.

[6] H. Silverman, "Harmonic univalent functions with negative coefficients," Journal of Mathematical Analysis and Applications, vol. 220, no. 1, pp. 283-289, 1998.

[7] H. Silverman and E. M. Silvia, "Subclasses of harmonic univalent functions," New Zealand Journal of Mathematics, vol. 28, no. 2, pp. 275-284, 1999.

[8] J. M. Jahangiri, "Coefficient bounds and univalence criteria for harmonic functions with negative coefficients," Annales Universitatis Mariae Curie-Skłodowska. Sectio A, vol. 52, no. 2, pp. 57-66, 1998.

[9] J. M. Jahangiri and H. Silverman, "Harmonic univalent functions with varying arguments," International Journal of Applied Mathematics, vol. 8, no. 3, pp. 267-275, 2002.

[10] S. Ruscheweyh, "Neighborhoods of univalent functions," Proceedings of the American Mathematical Society, vol. 81, no. 4, pp. 521-527, 1981. 